\title{
O Acervo Paleontológico da Universidade Federal de São Paulo - Campus Diadema
}

\author{
The Paleontological Collection from Universidade Federal de São Paulo - Campus Diadema
}

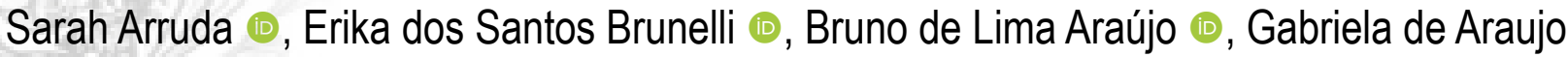 Pereira (), Suzany dos Santos Miranda $\odot$, Ana Luisa Vietti Bitencourt ()}

Universidade Federal de São Paulo, Diadema, São Paulo, SP, Brasil

E-mails: sarah.arruda18@gmail.com; erika.sbrunelli@gmail.com; bdelaraujo@gmail.com; gabi.pereiraa98@gmail.com; s.miranda@unifesp.br; ana.bitencourt@unifesp.br

\section{Resumo}

Os acervos paleontológicos são de grande importância para a pesquisa científica, extensão e ensino. Atualmente, existem onze acervos espalhados pelo estado de São Paulo, sendo o acervo da Universidade Federal de São Paulo (UNIFESP) - campus Diadema um dos mais recentes, iniciado por meio do Programa de Educação Tutorial (PET) em 2007, contendo 1030 exemplares. O presente trabalho tem como objetivo divulgar o acervo paleontológico da UNIFESP Diadema, descrever a sua formação, a sua constituição e seus públicos alvos. Apesar de novo, tem sido uma ferramenta relevante para diversos usos, como material de apoio para o ensino de graduação nas unidades curriculares de Paleontologia e Zoologia, com potencialidades à pesquisa e elaboração de trabalhos de conclusão de curso (TCC), além de atividades de extensão universitária, sendo estas essenciais no exercício formativo e do conhecimento paleontológico para a comunidade do município de Diadema, situada na região sul metropolitana de São Paulo.

Palavras-chave: Coleção paleontológica; Fósseis; Paleontologia

\section{Abstract}

The paleontological collections have a huge importance to scientific research, community outreach and education. Nowadays, there are eleven of these collections in the state of São Paulo and the Universidade Federal de São Paulo - campus Diadema collection's is one of the most recent. It started in 2007 with the Programa de Educação Tutorial (PET) and, currently, there are 1030 exemplars. This paper aims to disseminate the paleontological collection of UNIFESP Diadema, to describe its construction, constitution and its different target audiences. Despite being recent, the collection is a relevant tool for many uses, for example undergraduate education in the disciplines of Paleontology and Zoology, with potentialities to scientific research and undergraduate thesis and also to several university community outreach projects, essential for the dissemination and democratization of this knowledge in the community on the municipality of Diadema, located in the south metropolitan region of São Paulo.

Keywords: Paleontological Collection; Fossils; Paleontology

\section{Introdução}

Fósseis são exemplares que passaram por processos longos de alterações físicas, químicas e antrópicas (Ponciano et al., 2011). São considerados patrimônio da União pelos artigos 20 e 216 da Constituição Federal, a qual regula, entre outros aspectos, a não comercialização dos mesmos (Brasil, 1988). O patrimônio fossilífero tem grande importância para ações de preservação cultural e natural, englobando desde monumentos e sítios de valores arqueológicos e naturais, com formações físicas, biológicas ou geológicas consideradas excepcionais a geoparques mundiais da Unesco (Moreira, 2014; Rocha et al., 2017).

Em algumas situações, os fósseis são retirados de seu ambiente natural e alocados em acervos paleontológicos, sob a tutela de instituições de ensino, pesquisa ou em museus, a fim de preservá-los, estudá-los e/ou divulgálos (Kunzler et al., 2014). Estes acervos são de grande 
importância para a sociedade, seja pelo cunho científico, auxiliando no desenvolvimento de pesquisas, ou no âmbito histórico, pois são constituídos de fósseis que representam a história de vida do planeta, mostrando o passado dos seres vivos e dos ambientes da Terra (Diniz et al., 2015).

Outro aspecto importante relacionado aos acervos paleontológicos incluem a preservação de espécimes únicos. Trabalhos de campo e o transporte destes materiais possuem altos custos e demandam grandes esforços por parte dos pesquisadores e equipes envolvidas para seu resgate, já que eles podem ser grandes, pesados e/ou estarem localizados em regiões de difícil acesso. Portanto, o armazenamento dos fósseis em acervos contribui não somente para sua preservação enquanto registro da história da Terra, mas também possibilita a continuidade de seu estudo em laboratórios, valorizando o tempo e o dinheiro investido nos trabalhos de campo e/ou por outras instituições, concentrando esses materiais em um local de referência (Suarez \& Tsutsui, 2004; Kunzler et al., 2014). Nas regiões onde estão implementados, os acervos também podem ser uma ferramenta importante para a divulgação científica e inclusão social da população, auxiliando na educação científica e sendo um importante mecanismo pedagógico (Moreira, 2006). Bons acervos paleontológicos atraem mais pesquisadores para a instituição, que consequentemente são divulgados ao público, fazendo com que ela ocupe uma posição mais destacada e possa receber mais verbas, utilizando-as para melhorar a sua estrutura e serviços prestados (Kellner, 2005).

A divulgação do material paleontológico pode ser feita por meio de publicações ou apresentações em eventos científicos, além de ações educativas, através de exposições em museus ou em projetos de extensão, permitindo maior acesso e difusão de conhecimento para a população (Kunzler et al., 2014). Além disso, pode trazer benefícios socioeconômicos, como o de gerar emprego por meio do geoturismo (Ponciano et al., 2011). Portanto, a divulgação e democratização deste conhecimento mostra-se relevante para firmar os laços entre universidade e comunidade civil, considerando que tais ações colaboram para uma maior valorização e reconhecimento desses bens por parte da sociedade.

Atualmente, existem 11 instituições que abrigam acervos paleontológicos no estado de São Paulo, entre universidades e museus de ciências (Manzig, 2015). Dentre elas, encontra-se o acervo da Universidade Federal de São Paulo (UNIFESP) campus Diadema, que teve seu início em 2007, a partir da implementação do Programa de Educação Tutorial (PET) - Ciências Biológicas, pelo Edital Sesu-Mec n. 04/2007, como PET temático na área "Meio Ambiente e Uso Sustentável dos Recursos Naturais". Conduzida pela tutora Ana Luisa Vietti Bitencourt até 2016, a proposta teve como foco dar início às Coleções Científica e Didática nas áreas de Paleontologia e Palinologia, visando alicerçar, inicialmente, o ensino de graduação, com perspectivas para a pesquisa e a extensão, em um campus emergente.

As informações gerais sobre quantidade e composição de acervos paleontológicos em museus no Brasil ainda são muito escassas (Kellner, 2005), situação que também ocorre em universidades. Nesse sentido, é imprescindível que haja uma divulgação maior por parte destas instituições, incentivando a colaboração e a difusão dos exemplares para a população, principalmente da região em que está inserida. Portanto, o objetivo deste trabalho é apresentar o histórico da formação do acervo paleontológico da UNIFESP, sua constituição atual, sua importância e seus diferentes usos e públicos alvos.

\section{Material e Métodos}

\subsection{A Implementação do Acervo}

O acervo paleontológico da UNIFESP teve início pela aquisição de réplicas de fósseis para as aulas práticas do ensino de graduação dos cursos de Ciências Biológicas e Ciências Ambientais. Foi estruturado com o intuito de abranger o maior número de taxa, a fim de refletir a diversidade existente no passado, de maneira a proporcionar maior suporte para os estudantes compreenderem a evolução e a história da vida. Neste sentido, o acervo foi sendo gradativamente ampliado tanto a partir de coletas de exemplares fósseis em trabalhos de campo da unidade curricular de Paleontologia, quanto de projetos de pesquisa.

Nesse contexto, além do grupo PET, destacamse dois projetos que auxiliaram a promover a formação deste acervo: CNPq (Edital n” 32/2010) “Consolidação da Coleção Paleontológica do Laboratório de Paleoecologia e Ecologia de Paisagem da Universidade Federal de São Paulo (UNIFESP)-Campus Diadema", coordenado por Décio Luis Semensatto Junior; e CNPq (Edital MCT/ CNPq n" 023/2011) "Ampliação e consolidação da coleção paleontológica da Universidade Federal de São Paulo (UNIFESP), Campus Diadema", coordenado por Sandro Scheffler.

Somado ao material coletado em campo, alguns exemplares foram recebidos a partir de doações de instituições como o Instituto de Geociências da Universidade de São Paulo, Museu de Paleontologia Professor Antônio Celso de Arruda Campos e o Museu Nacional do Rio de Janeiro. Além disso, a aquisição de réplicas fósseis como material didático visou abranger um maior número de grupos taxonômicos, de estruturas esqueléticas completas em contextos geológicos e cronológicos diferentes. 


\subsection{A Organização do Acervo}

Há uma divisão em relação aos usos dos acervos universitários já descritos, sendo que eles podem ser majoritariamente científicos (p.ex., Lessa et al., 2018), didáticos (p.ex., Barbosa, 2000) ou com ambos os focos (p.ex., Duque \& Barreto, 2011). Alguns requisitos são necessários na construção de um acervo, como ter o registro da documentação em um livro tombo, manter a preservação e integridade das informações do registro, assim como a longevidade e a qualidade das informações asseguradas para pesquisas futuras (DOU, 2007; Carvalho, 2010). O acervo da UNIFESP possui ambos os focos, didático e pesquisa, sendo categorizado baseado no Sistema LUND (Sistema LUND, 2020), que considera agrupamentos nas categorias: paleovertebrados, paleoinvertebrados, microfósseis, paleobotânica e icnofósseis. Além disso, é estruturado a partir dos espécimes e grupos taxonômicos, tempos geológicos, unidades estratigráficas e referências bibliográficas.

O material cadastrado é tombado nas Coleções Científica ou Didática. A Coleção Científica $(\mathrm{Cc})$ está organizada em: Invertebrados (Cc-In), Vertebrados (Cc-Vt), Icnofósseis (Cc-Ic), Paleobotânica ( $\mathrm{Cc}-\mathrm{Pb})$ e Microfósseis $(\mathrm{Cc}-\mathrm{Mi})$. Esses exemplares são coletados em campo com as referências de local, data, coletor, formação geológica e dados de coordenadas geográficas. Já a Coleção Didática (Dd) agrupa um conjunto de réplicas paleontológicas e fósseis com informações incompletas a respeito da coleta e proveniência do material.

Para o armazenamento, metodologias usuais são utilizadas, como a limpeza e a preparação dos materiais, utilizando a pincelagem e raspagem por espátulas cirúrgicas odontológicas, sendo que no final cada amostra recebe um código de identificação. A identificação dos exemplares é efetuada, quando possível, de acordo com o nível taxonômico mais exclusivo, exceto para aqueles exemplares que estão inseridos no contexto da Biota Ediacarana. O registro é feito fisicamente na peça e digitalmente em um livro tombo com a sigla da instituição, seguido da categoria a qual pertence e pelo número geral do acervo, dado pela ordem de chegada, além de informações como local e data da coleta, quando existentes. Por fim, essas informações são reproduzidas em uma ficha que é anexada ao fóssil no local de armazenamento, permitindo a sua rápida identificação.

Após este procedimento, o material é armazenado em armários (Figura 1) correspondentes às Coleções Científica e Didática. As réplicas também passam pelo mesmo procedimento de tombamento, sendo alocadas na Coleção Didática.



Figura 1 Exemplo do armário da Coleção Científica, contendo os exemplares organizados. 


\subsection{A Divulgação do Acervo}

Levando em consideração a importância da divulgação científica, a difusão acerca deste acervo é feita por meio de exposições, extensão universitária, apresentações em eventos acadêmicos e por postagens em meio digital, através de mídias sociais como Instagram, Facebook e, principalmente, por vídeos no Youtube no canal "PET Biologia - UNIFESP".

\section{Resultados}

\subsection{O Acervo}

O acervo atualmente é composto por 1030 peças, organizadas nas Coleções Científica e Didática, representadas por 11 taxa, sendo 55 réplicas e 975 fósseis (Figura 2).

Os invertebrados correspondem ao grupo mais abundante, sendo constituídos por moldes e contramoldes. São 506 exemplares divididos em 8 taxa (Annelida, Arthropoda, Brachiopoda, Cnidaria, Echinodermata, Mollusca, Porifera e Biota Ediacarana). Assim sendo, a composição majoritária de taxa como Brachiopoda, com 221 fósseis, Mollusca com 174 fósseis e Echinodermata com 32 fósseis, ocorre devido às características geológicas dos locais de coleta e pelo próprio interesse da pesquisa. Os vegetais são o segundo grupo mais representativo, com 227 peças, abrangendo estruturas como folhas, troncos, moldes e contramoldes. Em seguida, os vertebrados estão representados pelos Chordata com 202 espécimes, possuindo diversas estruturas corporais parciais ou completas. Há 95 exemplares correspondentes aos registros de vestígios de atividades biológicas, agrupados nos icnofósseis. Algumas das peças presentes nas Coleções Didática e Científica estão ilustradas na Figura 3.

A Coleção Didática é composta por fósseis coletados durante as atividades de ensino e/ou doados por outras instituições, cujas informações de proveniência do material, muitas vezes, são incompletas. As réplicas adquiridas pelo PET também são cadastradas nesta categoria, sendo utilizadas tanto no ensino, para as atividades práticas das unidades curriculares de Paleontologia e Zoologia, quanto em eventos de extensão. Nesta coleção estão depositados 549 fósseis divididos em 8 taxa e 55 réplicas divididas em 7 taxa (Figura 4).

A Coleção Científica corresponde aos fósseis que foram coletados em projetos de pesquisa ou pelo PET, contendo informações completas sobre a origem das peças, como dados sobre a formação geológica, a posição estratigráfica, cronologia, coletor, local e data da coleta. Ela é composta por 426 fósseis, divididos em 9 taxa (Figura 5).

\subsection{Locais de Coleta}

As áreas de coleta da Coleção Científica (Figura 6) estão preferencialmente na Bacia do Paraná, Formações Corumbataí e Botucatu no estado de São Paulo, Furnas e Ponta Grossa no estado do Paraná e a Bacia de Taubaté, Formação Tremembé do estado de São Paulo.

Em relação a representatividade dos fósseis que possuem informações sobre a sua origem, destacam-se três bacias principais: a do Paraná, a do Araripe e a de Taubaté. Dentre estas, a Bacia do Paraná é a que apresenta o maior

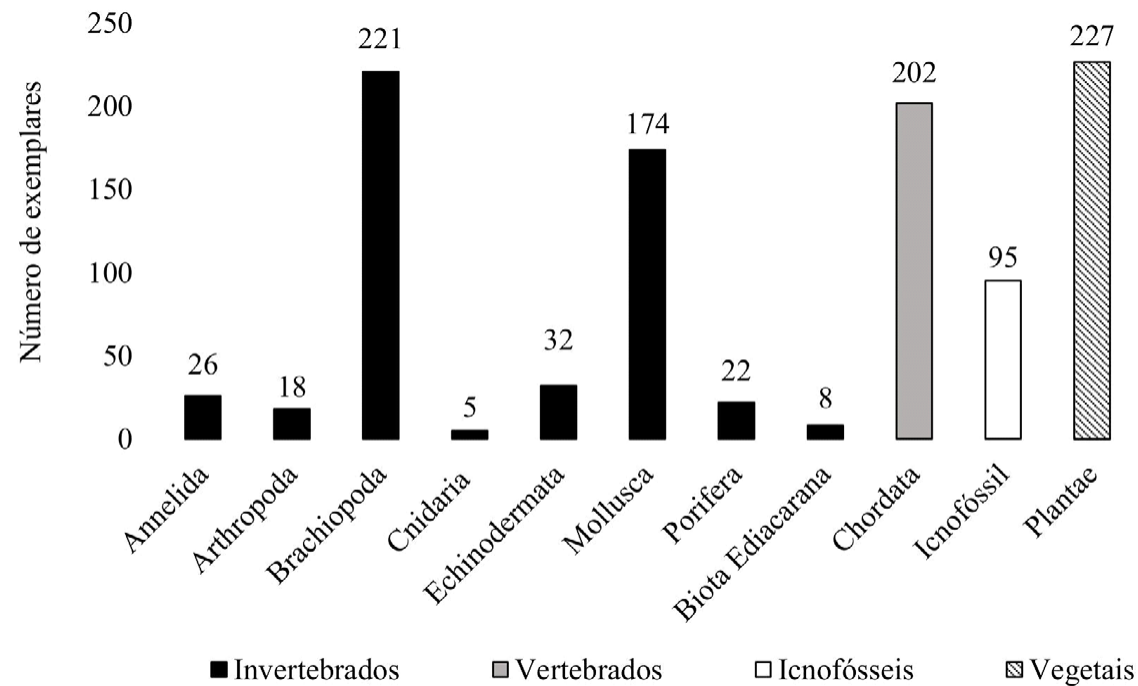

Figura 2 Distribuição de todos exemplares do acervo de acordo com os taxa presentes. 


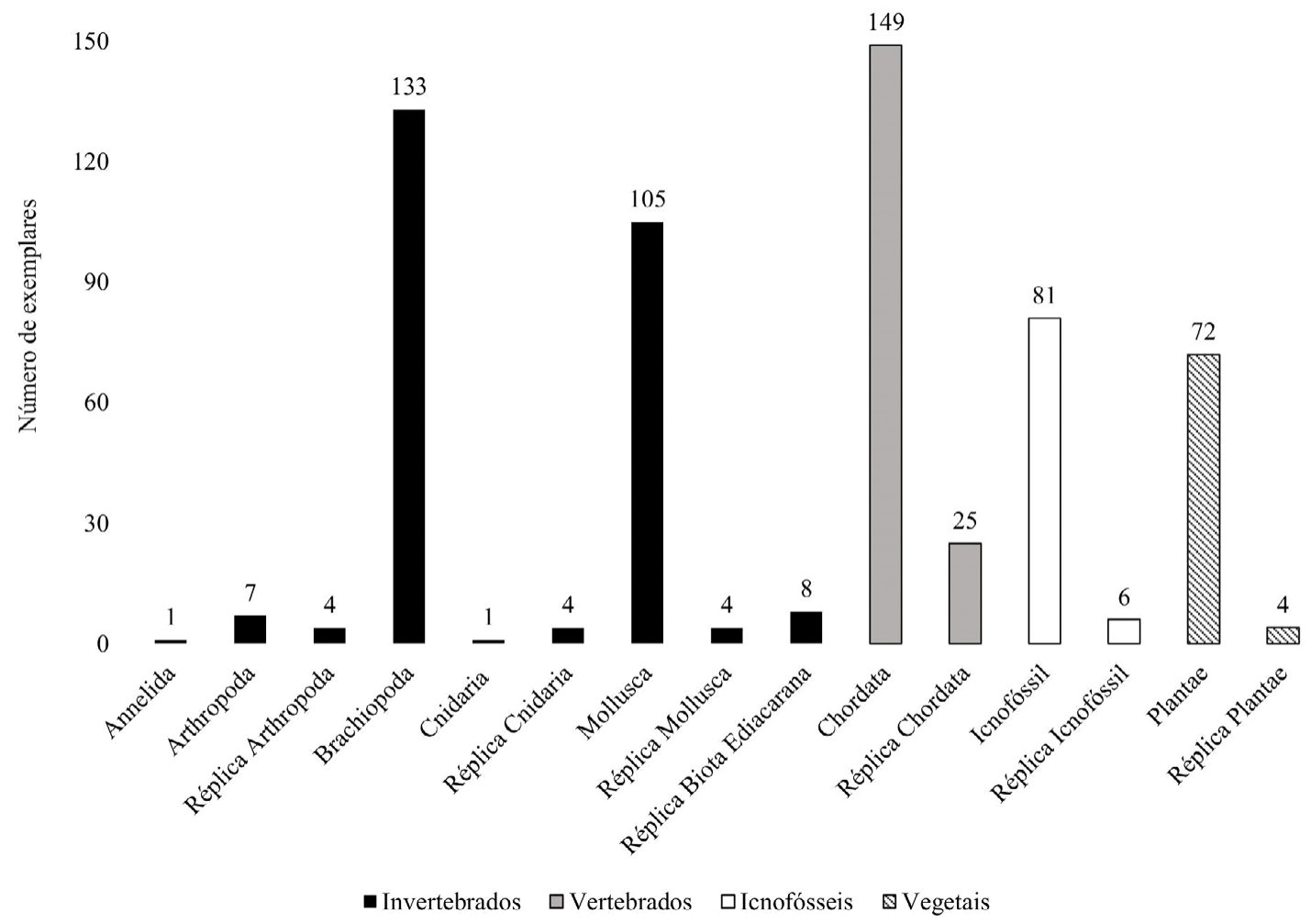

Figura 3 Distribuição dos taxa da Coleção Didática.


Figura 4 Exemplares presentes nas Coleções Científica (A-C) e Didática (D-F). A. Plantae (Fragmento Vegetal), B. Brachiopoda, C. Chordata (Actinopterygii), D. Icnofóssil, E. Plantae (tronco permineralizado) e F. Chordata (Pachycephalosaurus sp.). 


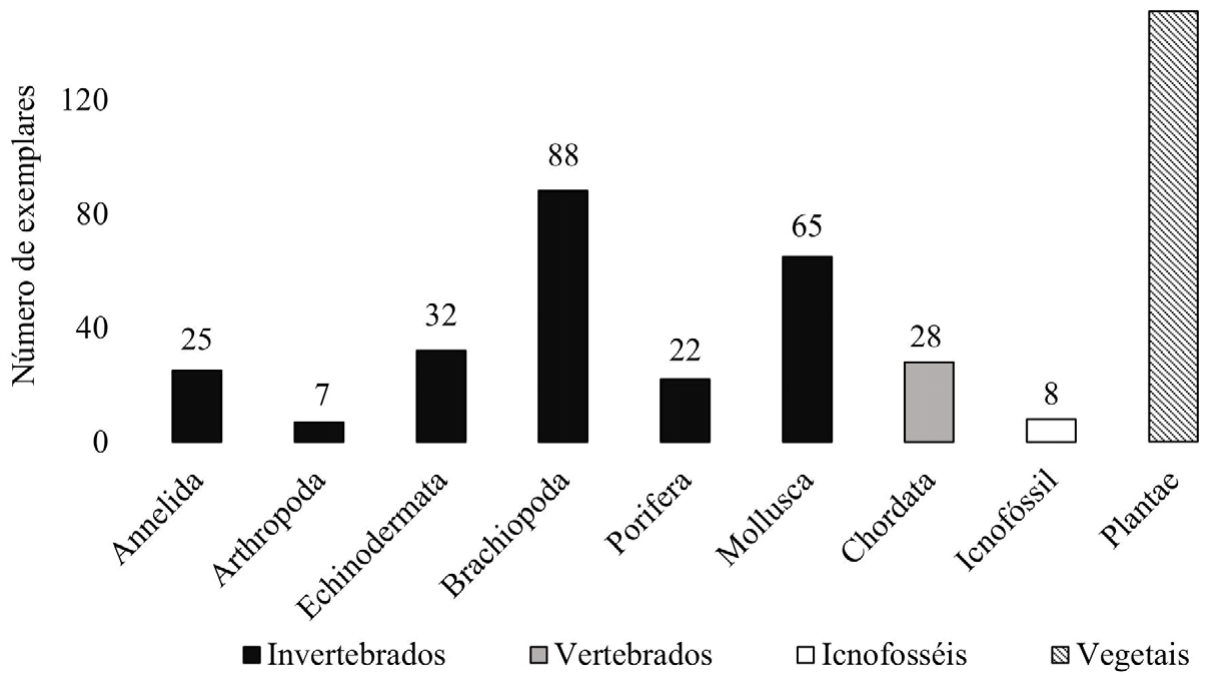

Figura 5 Distribuição dos taxa da Coleção Científica.

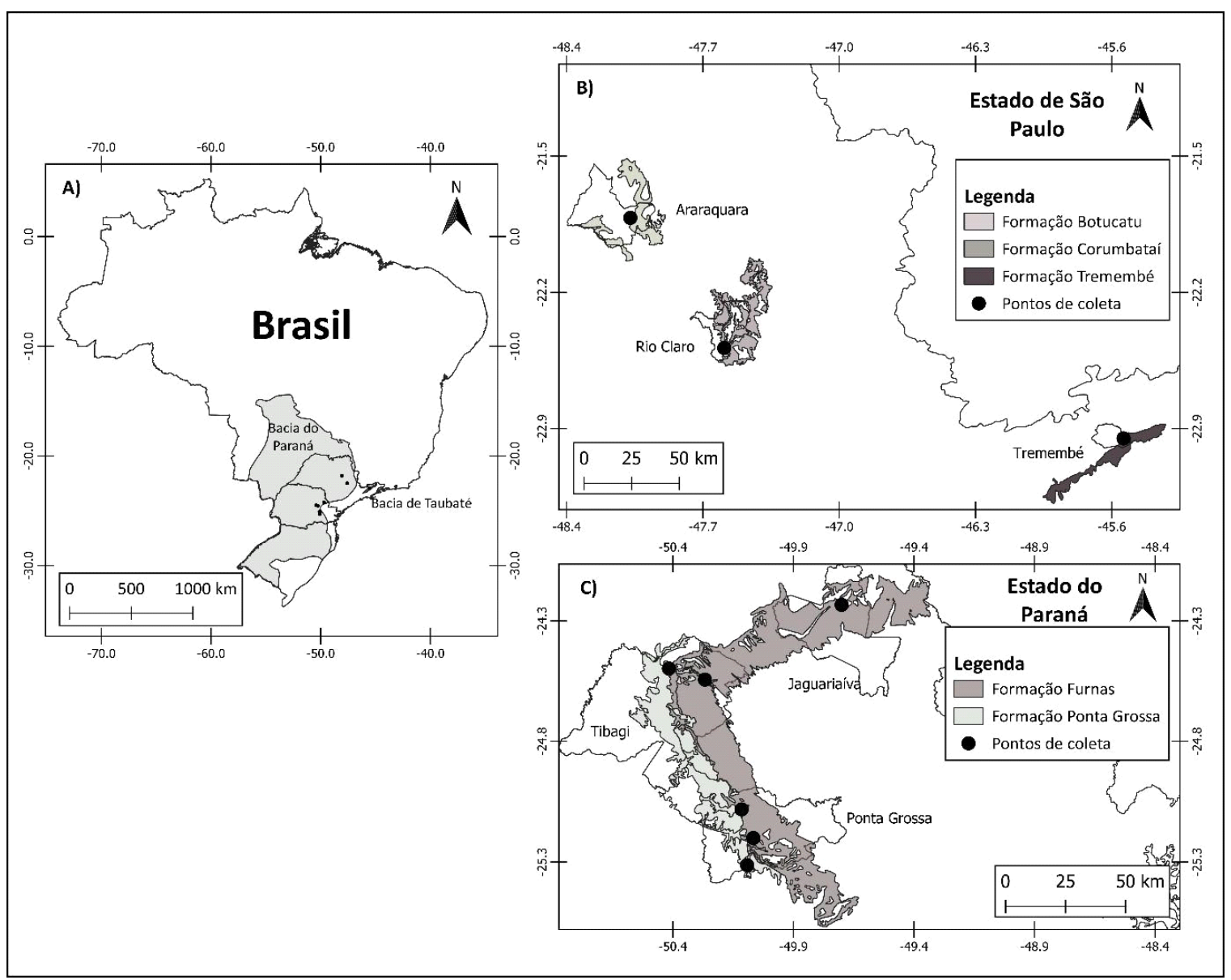

Figura 6 Localização das áreas de coleta dos fósseis da Coleção Científica. A. Localização geográfica das Bacias do Paraná e de Taubaté no território Brasileiro; B. Principais sítios de coletas no Estado de São Paulo; C. Principais sítios de coletas Estado do Paraná. Fonte: Serviço Geológico Brasileiro (SGB, 2020); Elaboração: Arruda (2020); software QGIS. 
número de exemplares, provenientes das formações Ponta Grossa, Furnas e Corumbataí. Do total de 975 fósseis, 652 possuem informações sobre sua origem (Figura 7).

\subsection{Divulgação do Acervo}

Em relação a divulgação do acervo, o PET conta com um setor de audiovisual, o qual possui um canal no YouTube, onde estão disponíveis diversos vídeos de atividades realizadas pelo grupo (Arruda et al., 2018a). Um exemplo é o vídeo "Coleções Biológicas: paleontologia e palinologia" (PET Biologia - UNIFESP, 2016), cuja temática abordada explica o que é e qual a importância das coleções Paleontológicas e Palinológicas. O vídeo "O que o subgrupo da Paleo faz?” (PET Biologia - UNIFESP, 2019) relata as principais atividades realizadas com o acervo, mencionando as oficinas realizadas, o processo de curadoria e exibição de que forma os exemplares são organizados nos armários.

Desde sua criação, a exposição de exemplares fósseis do acervo foi levada em vários eventos de extensão em parceria com a prefeitura municipal de Diadema e em eventos do campus de Diadema (Figura 8). Entre os destaques está o "Experimentando a Ciência", que ocorreu entre 2007 e 2010, atingindo neste período cerca de dez mil pessoas, entre escolas e público em geral. A exposição também esteve presente no "Festival do Livro" (2011, 2012, 2013), evento público realizado na Praça da Moça e no Teatro Clara Nunes no centro da cidade, atingindo milhares de participantes. Outro grande evento foi o "Mês do Meio Ambiente em Diadema", que visou ampliar a Educação Ambiental do público em geral no Shopping Praça da Moça em 2017 (Portal ABC do ABC, 2017).

Outras ações extensionistas envolveram oficinas com o acervo, como "Hoje é Dia de Paleontologia", que objetivou promover a interação com escolas do bairro Eldorado-Diadema, introduzindo o conhecimento das Ciências Naturais através da Paleontologia e das Ciências da Terra, por meio de atividades lúdicas e interativas. O intuito foi despertar nas crianças o interesse pela educação ambiental com relação à história evolutiva do planeta Terra, incluindo a evolução da vida, preservação e evolução dos ambientes terrestres e marinhos. Algumas atividades lúdicas foram realizadas para introduzir o trabalho do paleontólogo como "Detetive do Passado", que envolveu desde a descoberta até análise dos fósseis, através de mini escavação em caixa de areia, confecção de réplicas, pinturas em desenhos de fósseis, moldes de argila e jogos. Essa atividade envolveu centenas de crianças das séries iniciais do ensino básico da escola Novo Eldorado (UNIFESP, 2016).

\section{Discussão e Considerações Finais}

O principal uso do acervo é voltado para o ensino de Paleontologia na graduação, nos cursos de Ciências Ambientais, Biológicas e, mais recentemente, para o curso de Licenciatura em Ciências. Além disso, este material também tem sido utilizado no ensino de Zoologia em

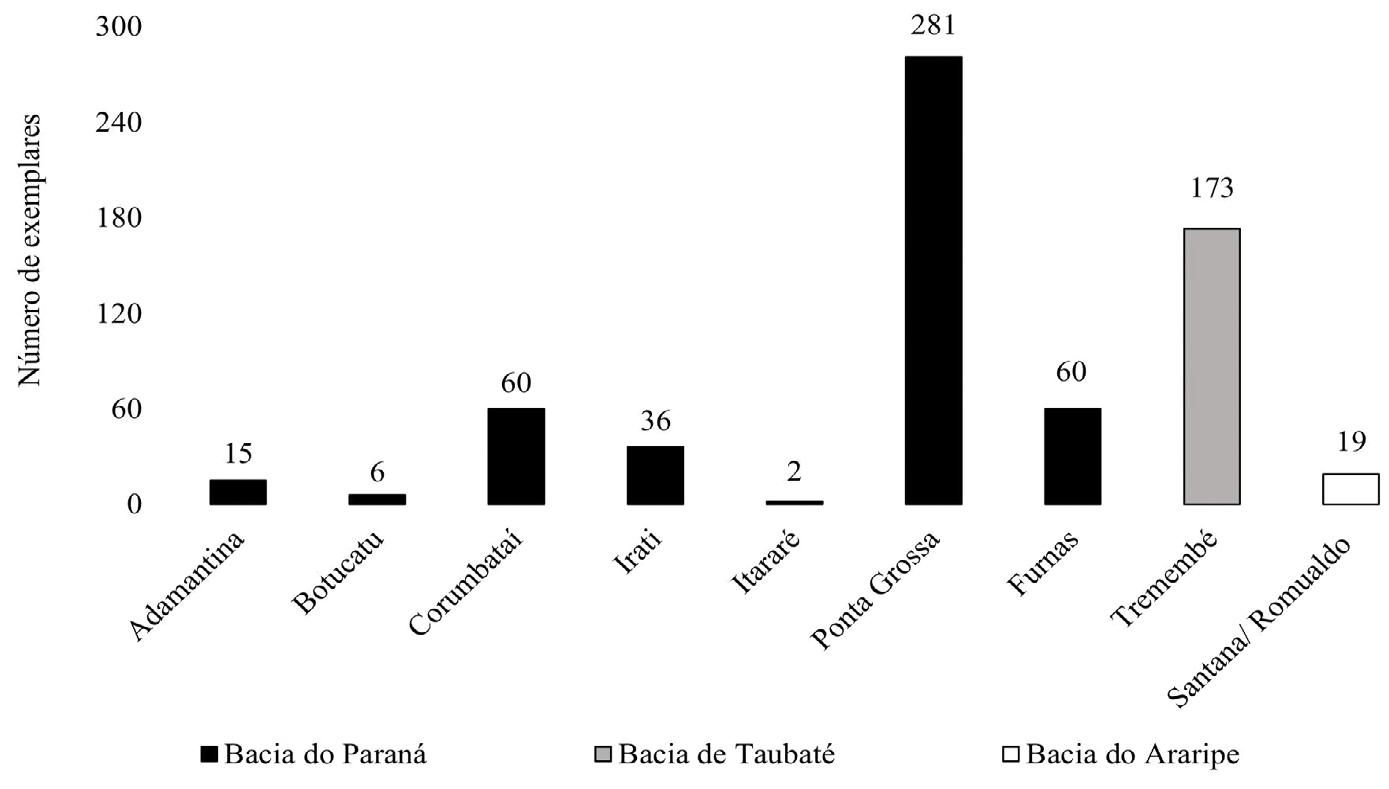

Figura 7 Representatividade das formações geológicas do acervo. 


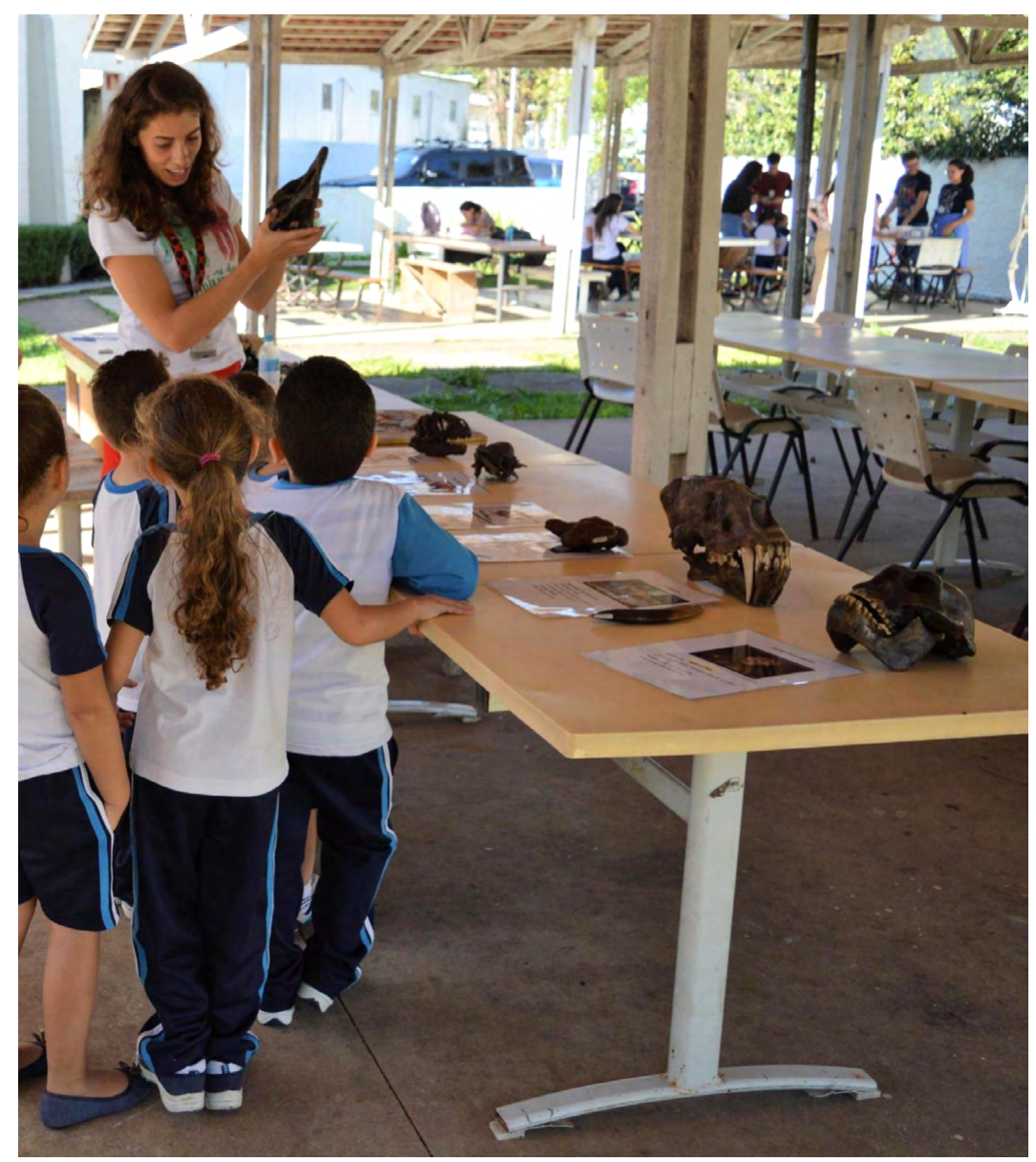

Figura 8 Exemplo de exposição do acervo em uma atividade de extensão.

suas aulas práticas, com foco investigativo em padrões evolutivos de grandes grupos taxonômicos de invertebrados e vertebrados.

As coletas são feitas por estudantes da unidade curricular de Paleontologia, sendo parte do componente pedagógico e prático no ensino da graduação. Sendo assim, a composição do acervo está diretamente relacionada ao acesso às formações geológicas mais próximas ao campus Diadema. Dessa forma, a maioria dos fósseis do acervo são provenientes dos sítios paleontológicos da Bacia do Paraná, Formações Irati e Corumbataí, na região de Rio Claro, e na Bacia de Taubaté, Formação Tremembé, no município de Tremembé, SP.

Os exemplares representam os períodos do Devoniano e Permiano (para a Bacia do Paraná) e do período Terciário (para a Bacia de Taubaté) no estado de São Paulo. Neste contexto, os fósseis de ambiente marinho são os mais abundantes, dentre eles os invertebrados (Mollusca e Brachiopoda) para a Bacia do Paraná. Além destes, também há fragmentos vegetais, vértebras e esqueletos de peixes da Bacia de Taubaté, configurando o conjunto de fatores que influenciaram a composição do acervo.

Dentre os grupos estudados do acervo, os invertebrados destacam-se como o taxon mais abundante (506 exemplares), sendo tema de um trabalho de conclusão de curso realizado com crinóides e que posteriormente resultou em um artigo (Francisco, 2013; Scheffler et al., 2019). Ademais, dados parciais do acervo foram apresentados em eventos científicos como congressos, simpósios e encontros regionais promovidos pela Sociedade Brasileira de Paleontologia e pela UNIFESP (p.ex., Coa et al., 2012; Francisco et al., 2013; Chaves et al., 2014; Silva et al., 2016; Arruda et al., 2018b). O acervo oferece um grande potencial para o desenvolvimento de pesquisa envolvendo processos tafonômicos, em especial para alguns grupos de vertebrados como mesossaurídeos (Formação Irati) ou peixes (Formação Tremembé), além de grupos vegetais como troncos fósseis (Formação Tremembé).

Em adição aos eventos científicos e dos usos didáticos, o acervo é amplamente utilizado em exposições e 
em atividades de extensão junto à comunidade local, sendo uma ferramenta importante para a educação científica na região. Como um aspecto formativo e de inclusão social, a divulgação científica é importante para viabilizar que cada cidadão tenha a oportunidade de adquirir conhecimento básico sobre o papel da Paleontologia na ciência e em seu funcionamento (Moreira, 2006). Além disso, esta aproximação universidade-comunidade é importante para que as pessoas compreendam e valorizem o patrimônio paleontológico e da ciência como um todo.

Neste sentido, o acervo, apesar de ter um número restrito de exemplares, é capaz de atender várias demandas, sejam elas para fins didáticos nas disciplinas da graduação, de difusão para a comunidade ou científicos. No entanto, para este último, ainda são necessárias novas coletas para o desenvolvimento de pesquisas visando uma maior representatividade taxonômica e geológica. Deste modo, o acervo da UNIFESP vem contribuindo para a democratização do acesso à cultura, por meio da divulgação da ciência, sensibilizando sobre a preservação do patrimônio fossilífero brasileiro.

\section{Agradecimentos}

Os autores agradecem ao Programa de Educação Tutorial (Sesu-MEC) pelas bolsas e a verba custeio para o desenvolvimento e a manutenção do acervo; ao CNPq pelos financiamentos obtidos (Edital 32/2010 e Edital MCT/ CNPq $n^{\circ} 023 / 2011$ ) e a todos que contribuíram e atuaram na formação do acervo.

\section{Referências}

Arruda, S.; Fernandez, M.S.; Cruz, G.V.; Bartasevicius, V.T.; Pereira, T.M.; Jaimes, J.N.; Silva, M.M.; Barbosa, B.C; Pereira, G.A.; Park, P.S.H.; Perez, I.C.S.; Magalhaes, T.J.; Brunelli, E.S.; Miranda, S.S.; Silva, J.A.S.; Nogueira, M.P.; Quagliano, J.A.; Catai, N.R.; Araújo, B.L.; Baldini, A.; Santos, F.E.C.; Bianco, A.A.G.; Rodrigues, E. \& Bitencourt, A.L.V. 2018a. Projeto Audiovisual PET: Uma Nova Abordagem Para Divulgação de Conhecimento Científico. In: CONGRESSO ACADÊMICO DA UNIFESP - CAMPUS DIADEMA, 4, Diadema, 2018. Anais do IV Congresso Acadêmico da UNIFESP, Diadema, UNIFESP, p. 1154-1155.

Arruda, S.; Brunelli, E. S.; Pereira, G. A.; Araújo, B. L.; Miranda, S. S.; Magalhaes, T. J.; Quaglio, F. \& BITENCOURT, A. L. V. 2018b. O Acervo da Coleção Paleontológica da Universidade Federal de São Paulo - Campus Diadema - SP. In: REUNIÃO ANUAL DA SOCIEDADE BRASILEIRA DE PALEONTOLOGIA - REGIONAL SP, 19, Campinas, 2018. Resumos Paleo Sp 2018, Campinas, SBP, p. 54.

Barbosa, L.M. 2000. Importância de uma coleção mineralógicapetrográfica-paleontológica e o acervo do Laboratório de Geociências e Geologia da UEFS. Sitientibus, 23(1): 9-18.
Brasil. 1988. Constituição da República Federativa do Brasil: promulgada em 5 de outubro de 1988. Disponível em: <http:// www.planalto.gov.br/ccivil_03/constituicao/constituicao. htm>. Acesso em: 15 out. 2019.

Carvalho, I.S. 2010. Curadoria Paleontológica. In: CARVALHO I.S. (Ed.). Paleontologia. Rio de Janeiro: Interciência, p. 397-412.

Chaves, M.N.; Cotrim, E.; Melo, E.D.; Ribeiro, M.T.; Bitencourt, A.L.V. \& Nunes, F.R.C. 2014. Curadoria da Coleção Paleontológica da Unifesp, Campus Diadema Grupo Pet Ciências Biológicas. In: REUNIÃO ANUAL DA SOCIEDADE BRASILEIRA DE PALEONTOLOGIA - REGIONAL SP, 15, São Paulo, 2014. Livro De Resumos, São Paulo, SBP, p. 21.

Coa, L.L. et al. 2012. Implantação e ampliação da coleção paleontológica do Campus Diadema, Unifesp. In: SIMPÓSIO BRASILEIRO DE PALEOINVERTEBRADOS, 1, Bauru, 2012. Paleontologia em Destaque, Rio de Janeiro, SBP, p. 37.

Diniz, D.; Ioewenstein, H. \& Dentzien-Dias, P.C. 2015. Curadoria do acervo paleontológico da Universidade Federal do Rio Grande. Revista Museologia \& Interdisciplinaridade, 4(7): 257-267.

DOU. 2007. Diário Oficial da União. Instrução Normativa 160. Ministério do Meio Ambiente, Instituto Brasileiro do Meio ambiente e Recursos Renováveis, 82(1): 404-405.

Duque, R.R.C. \& Barreto, A.M.F. 2011. Procedimentos de curadoria em parte da coleção científica de fósseis do Departamento de Geologia/CTG/UFPE. In: CONGRESSO DE INICIAÇÃO CIENTÍFICA DA UFPE, 19, Recife, 2011. Resumos expandidos, Recife, UFPE, p. 1-5.

Francisco, A.P.S. 2013. Equinodermas Pedunculados do Devoniano da Bacia do Paraná, Estados do Paraná e Goiás, Brasil. Trabalho de Conclusão de Curso (Ciências Biológicas), Universidade Federal de São Paulo, Diadema, 61 p.

Francisco, A.P.S.; Oliveira, F.R.F.; Kukimodo, I.; Coa, L.L.; Franco, R.R.A.; Scheffler, S.M. \& Bitencourt, A.L.V. 2013. Implementação das Pesquisas Paleontológicas da UNIFESP - Campus Diadema. In: CONGRESSO DE INICIAÇÃO CIENTÍFICA - PIBIC, 21, São Paulo, 2013. Anais do XXI Congresso de Iniciação Científica - PIBIC, São Paulo, UNIFESP, p. 750.

Kellner, A.W.A. 2005. Museus e a Divulgação Científica no Campo da Paleontologia. Anuário do Instituto de Geociências, 28(1): 116-130.

Kunzler, J.; Novaes, M.G.L.; Machado, D.M.C. \& Ponciano, L.C.M.O. 2014. Coleções Paleontológicas Como Proteção do Patrimônio Científico Brasileiro. In: SEMINÁRIO INTERNACIONAL CULTURA MATERIAL E PATRIMÔNIO DE C\&T, 3, Rio de Janeiro, 2014. Anais, Rio de Janeiro, MAST, p. 385-407.

Lessa, C.M.B.; Gomes, V.S.; Baleeiro, E.M.G.; Silva, K.O. \& Dantas, M.A.T. 2018. Coleção Científica de Fósseis do Laboratório de Ecologia e Geociências da Universidade Federal da Bahia (IMS/CAT): Acervo e Técnicas de Curadoria. Anuário do Instituto de Geociências, 41(2): 117-122. 
Manzig, P.C. 2015. Museus De Paleontologia No Brasil E A Paleontologia Nos Museus Brasileiros. Programa em PósGraduação em Divulgação Científica e Cultural, Universidade Estadual de Campinas, Dissertação de Mestrado, 200 p.

Moreira, I.C. 2006. A inclusão social e a popularização da ciência e tecnologia no Brasil. Inclusão social, 1(2): 11-16.

Moreira, J.C. 2014. Geoturismo e Interpretação Ambiental. Ponta Grossa, SciELO-Editora UEPG. 157 p.

PET Biologia - UNIFESP. 2016. Coleções Biológicas: paleontologia e palinologia. (12m43s). Disponível em: <https://youtu.be/ uFK-LpoHA-I $>$. Acesso em: 10 set. 2020.

PET Biologia - UNIFESP. 2019. O que o subgrupo da paleo faz? (2m39s). Disponível em: $<$ https://youtu.be/VeHJumHUb6g $>$. Acesso em: 10 set. 2020.

Ponciano, L.C.M.O.; Castro, A.R.S.F.; Machado, D.M.C.; Fonseca, V.M.M. \& Kunzler, J. 2011. Patrimônio geológicopaleontológico in-situ e ex-situ: definições, vantagens, desvantagens e estratégias de conservação. In: CARVALHO, I.S.; SRIVASTAVA, N.K.; JUNIOR, O.S. \& LANA, C.C. (Eds.). Paleontologia: Cenários de Vida. Editora Interciência, p. 853-869.

Portal ABC do ABC. 2017. Exposição de Fósseis e Plantas no Shopping Praça da Moça. Disponível em: <https://www. abcdoabc.com.br/diadema/noticia/exposicao-fosseis-plantasshopping-praca-moca-51812>. Acesso em: 29 set. 2020.

Rocha, L.C.; Ferreira, A.C. \& Figueiredo, M.A. 2017. A Rede Global de Geoparques e os desafios da integração dos Geoparques Brasileiros. Caderno de Geografia, 27(2): 271292.
Scheffler, S.M.; Francisco, A.P.S. \& Bosetti, E.P. 2019. Sobre os Equinodermos Devonianos da Bacia do Paraná: Mudanças Faunísticas e Implicações para a Utilidade Bioestratigráfica. Anuário do Instituto de Geociências, 40(3): 102-116.

SBG. 2020. Serviço Geológico Brasileiro. Dados, informações e produtos do Serviço Geológico do Brasil. Disponível em: $<$ http://geosgb.cprm.gov.br/geosgb/sobre_geosgb.html $>$. Acesso em: 01 dez. 2020.

Silva, P.D.; Brunelli, E.S.; Clerici, G.P.; Rodrigues, F.C.; Neto, V. C.; Morais, E.F.; Franzolin, G.N.; Magalhaes, T.J.; Junior, J.M.A.; Soliani, A.G.; Silva, L.R. \& Bitencourt, A.L.V. 2016. Grupo PET Ciências Biológicas: Hoje é Dia de Paleontologia. In: CONGRESSO ACADÊMICO, 2, São Paulo, 2016. Anais do II Congresso Acadêmico Unifesp, São Paulo, UNIFESP, p. 922.

Sistema LUND. 2020. Catálogo de fósseis. Disponível em: $<$ http:// sistemalund.com.br/index.php>. Acesso em: 29 set. 2020.

Suarez, A.V. \& Tsutsui, N.D. 2004. The Value of Museum Collections for Research and Society. BioScience, 54(1): 66-74.

UNIFESP. 2016. Universidade Federal de São Paulo. Oficina de Fósseis: Hoje é Dia de Paleontologia é realizada no Campus Diadema. Disponível em: <https://www.unifesp.br/reitoria/ dci/noticias-anteriores-dci/item/2161-oficina-de-fosseis-hojee-dia-de-paleontologia-e-realizada-no-campus-diadema $>$. Acesso em: 29 set. 2020.

\section{Como citar:}

Arruda.; S.; Brunelli, E.S.; Araújo, B.L.; Pereira, G.A.; Miranda, S.S. \& Bitencourt, A.L.V. 2021. O Acervo Paleontológico da Universidade Federal de São Paulo - Campus Diadema. Anuário do Instituto de Geociências, 44: 35587. DOI 1982-3908_2021_44_35587 